\title{
Perfil isotópico da fauna associada ao estuário interno do Rio Paraíba do Sul, norte do estado do Rio de Janeiro, Sudeste do Brasil
}

Esse estudo caracteriza o perfil isotópico de nitrogênio (d15N) e carbono (d13C) de crustáceos (Callinectes bocourti e Macrobrachium acanthurus) e peixes (Lycengraulis grossidens, Anchoviella lepidentostole, Leporinus copelandii, Genidens genidens e Centropomus undecimalis) que fazem parte da fauna associada ao estuário interno do Rio Paraíba do Sul. ANOVA demonstrou diferenças significantes entre as espécies em relação ao perfil isotópico de nitrogênio ( $F=38,2$, g.l.= $6, p<0.0001)$ e carbono ( $F=24,3, g . I .=6, p<0.0001)$. Genidens genidens se destaca das demais espécies com os valores mais pesados de d15N e d13C, enquanto $\mathrm{M}$. acanthurus apresentou os valores mais leves para ambos os isótopos. Os valores de d15N permitiram agrupar C. bocourti, M. acanthurus, A. lepidentostole $\mathrm{e}$ L. copelandii com os valores mais leves, seguido de L. grossidens e C. undecimalis, e depois G. genidens, destacada das demais espécies com a média mais pesada de d15N. Em relação a d13C, os valores mais leves se referem ao grupo formado por M. acanthurus, L. copelandii e C. undecimalis, depois C. bocourti com valor intermediário, e L. grossidens, A. lepidentostole e G. barbus com os valores mais pesados de d13C. Os dados desse estudo são os primeiros registros do perfil isotópico desses animais para a região e servirão de referência para estudos futuros.

\section{Isotopic profile of the fauna associated with the inner estuary of the Paraíba do Sul River, northern Rio de Janeiro state, Southeaster Brazil}

This study characterizes the isotopic profile of nitrogen (d15N) and carbon (d13C) of crustaceans (Callinectes bocourti and Macrobrachium acanthurus) and fish (Lycengraulis grossidens, Anchoviella lepidentostole, Leporinus copelandii, Genidens genidens and Centropomus undecimalis) from the fauna associated with the inner estuary of the Paraiba do Sul River. ANOVA demonstrated significant differences among species regard to the nitrogen $(F=38.2, \mathrm{gl}=6, \mathrm{p}<0.0001)$ and carbon $(F=24.3, g l=6, p<0.0001)$ isotopic profile. Genidens genidens stands out from the other species with the heaviest values of $d 15 \mathrm{~N}$ and $d 13 \mathrm{C}$, while $M$. acanthurus presented the lightest values for both isotopes. The $\mathrm{d} 15 \mathrm{~N}$ values allowed to group $\mathrm{C}$. bocourti, $\mathrm{M}$. acanthurus, A. lepidentostole and $\mathrm{L}$. copelandii with the lightest values, followed by L. grossidens and C. undecimalis, and then G. genidens, highlighted from the other species with the heavier d15N. In relation to d13C, the lightest values refer to the group formed by M. acanthurus, L. copelandii and C. undecimalis, then C. bocourti with intermediate value, and L. grossidens, A lepidentostole and G. genidens with heavier $\mathrm{d} 13 \mathrm{C}$ values. The data from this study are the first records of the isotopic profile of these animals for the region and will serve as a reference for future studies.

Keywords: Stable isotopes; Crustaceans; Fish; Estuary.

Topic: Notas Científicas

Reviewed anonymously in the process of blind peer.
Received: 06/10/2020

Approved: 28/11/2020
Ana Paula Madeira Di Beneditto iD

Universidade Estadual do Norte Fluminense Darcy Ribeiro, Brasil http://lattes.cnpq.br/8402221071942314

http://orcid.org/0000-0002-4248-9380 anapaula@uenf.br
Referencing this:

BENEDITTO, A. P. M.. Perfil isotópico da fauna associada ao estuário interno do Rio Paraíba do Sul, norte do estado do Rio de Janeiro, Sudeste do Brasil. Revista Ibero Americana de Ciências Ambientais, v.11, n.6, p.747-753, 2020. DOI: http://doi.org/10.6008/CBPC2179$\underline{6858.2020 .006 .0060}$

DOI: 10.6008/CBPC2179-6858.2020.006.0060 


\section{INTRODUÇÃO}

A bacia do Rio Paraíba do Sul faz parte da região hidrográfica do Leste brasileiro. Este rio tem uma bacia de drenagem com cerca de $56.500 \mathrm{~km}^{2}$, estendendo-se por áreas naturais, urbanizadas, industriais e rurais do sudeste do Brasil, que aportam matéria orgânica, sedimento e efluentes para a região costeira (KRUGER et al., 2003). Na parte inferior da bacia do Rio Paraíba do Sul, no estuário interno, nota-se um conjunto faunístico caracterizado pela mistura de espécies dulcícolas, marinhas e estuarinas, com animais residentes e temporários que incluem espécies anádromas e anfídromas que migram para atendimento de suas atividades reprodutivas e/ou alimentares (BIZERRIL et al., 2001). Os estudos mais abrangentes sobre a fauna associada ao estuário interno do Rio Paraíba do Sul tratam de inventários da ictiofauna (ARAÚJO, 1996; BIZZERIL et al., 2001). Dessa forma, há uma lacuna de informações sobre aspectos relacionados a ecologia da fauna local.

Isótopos estáveis de nitrogênio $\left(\delta^{15} \mathrm{~N}\right)$ e carbono $\left(\delta^{13} \mathrm{C}\right)$ são marcadores químicos utilizados em estudos que tratam da ecologia alimentar dos animais (BENEDITTO et al., 2018; GATTS et al., 2020). O perfil isotópico determinado nos tecidos dos animais deriva da combinação das fontes alimentares consumidas e do fracionamento isotópico nos tecidos corporais, refletindo o que foi assimilado após os processos de digestão e excreção (FRY, 2008). A análise da composição isotópica complementa dados sobre a ecologia alimentar dos animais e permite predizer a sua posição trófica e a origem das suas fontes alimentares (FRY, 2008). Embora ambos os isótopos aumentem gradualmente seus valores com o aumento dos níveis tróficos, o fator de enriquecimento para $\delta^{15} \mathrm{~N}$ é mais óbvio quando comparado a $\delta^{13} \mathrm{C}$. Este último isótopo estável é frequentemente utilizado para indicar a origem das fontes de carbono para a dieta, como por exemplo fontes costeiras vs. oceânicas, pelágicas vs. bentônicas ou aquáticas vs. terrestres (PETERSON et al., 1987).

Esse estudo caracteriza o perfil isotópico de nitrogênio $\left(\delta^{15} \mathrm{~N}\right)$ e carbono $\left(\delta^{13} \mathrm{C}\right)$ de espécies de crustáceos e peixes que fazem parte da fauna associada ao estuário interno do Rio Paraíba do Sul, próximo à sua foz principal, e compara esse perfil com o conhecimento prévio sobre o hábito e modo de alimentação preferencial desses animais. Devido à falta de informações sobre a ecologia alimentar da fauna local, os dados apresentados preencherão essa lacuna do conhecimento e servirão de referência para estudos futuros, pois é a primeira vez que o perfil isotópico desses animais é descrito para a região.

\section{RELATO}

Os crustáceos ( $n=2$ espécies) e peixes ( $n=5$ espécies) analisados foram coletados no estuário interno do Rio Paraíba do Sul com a colaboração de pescadores locais que utilizam rede de espera (peixes) e armadilhas de fundo (crustáceos) (Figura 1). Os animais foram capturados a partir de eventos de pesca praticados em 10 de março de 2011.

Após a captura, os indivíduos foram mantidos sob refrigeração e levados ao laboratório para identificação taxonômica (espécie), medida de tamanho corporal $(0.1 \mathrm{~cm})$ e peso total $(0.1 \mathrm{~g})$, e retirada de amostra de tecido muscular para análise do perfil isotópico. O músculo abdominal dos crustáceos e o músculo 
lateral dorsal dos peixes foram removidos de cada indivíduo e armazenados separadamente em frascos estéreis e secos, congelados $\left(-20^{\circ} \mathrm{C}\right)$, liofilizados por 48 horas e homogeneizados em grau e pistilo. Amostras de 0,4 mg de músculo (peso seco) de cada indivíduo foram utilizadas para analisar a composição isotópica de nitrogênio e carbono.

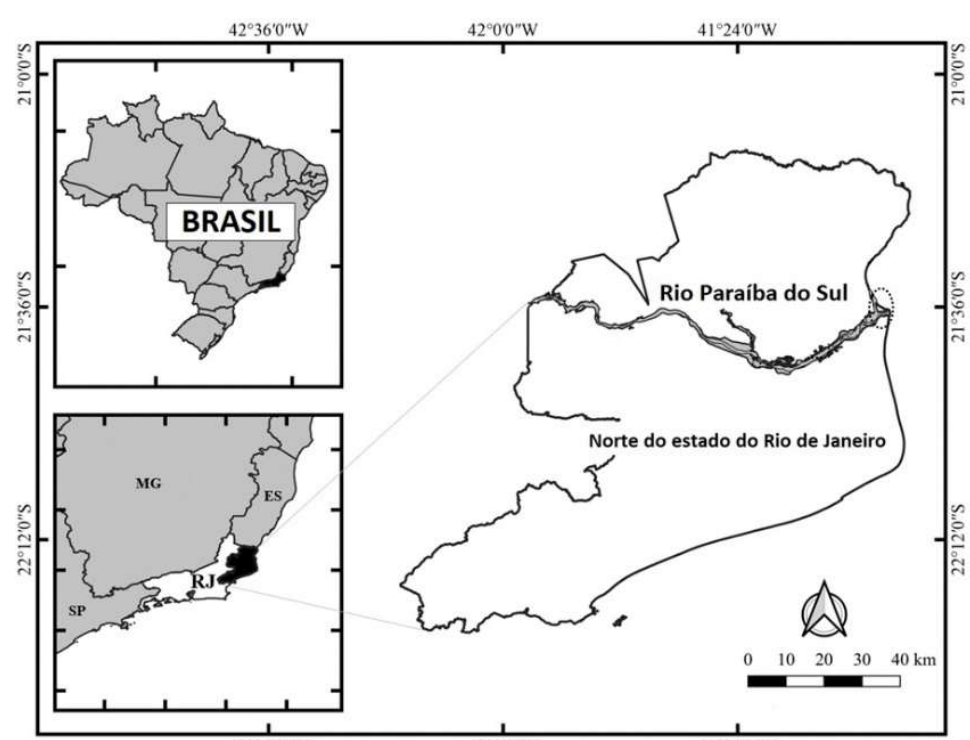

Figura 1: Mapa do Brasil e do estado do Rio de Janeiro com destaque para a costa norte do estado, onde se localiza o estuário interno do Rio Paraíba do Sul (círculo tracejado).

A composição isotópica do músculo dos indivíduos foi determinada em espectrômetro de massa Delta V Advantage (Thermo Scientific, Alemanha) acoplado ao analisador elementar no Laboratório de Ciências Ambientais da Universidade Estadual do Norte Fluminense Darcy Ribeiro. Os valores de referência usados nas análises de carbono foram Pee Dee Belemnite (PDB), e para nitrogênio o $N_{2}$ atmosférico. As amostras foram analisadas juntamente com brancos analíticos e padrões analíticos de ureia (IVA Analysentechnik-330802174; $\mathrm{CH}_{4} \mathrm{~N}_{2} \mathrm{O} \mathrm{Mw}=60, \mathrm{~N}=46 \%, \mathrm{C}=20$ \%) com composições isotópicas certificadas $\left(\delta^{15} \mathrm{~N}=-0,73 \%\right.$ e $\delta^{13} \mathrm{C}=-39,89 \%$ \%). O controle analítico foi realizado a cada 10 amostras, usando padrão isotópico certificado (Elemental Microanalysis Protein Standard OEA: $\delta^{15} \mathrm{~N}=+5,94 \%$ e $\delta^{13} \mathrm{C}=-26,98 \%$ ). A reprodutibilidade analítica foi baseada em triplicatas a cada 10 amostras: $\pm 0,3 \%$ para $\delta^{15} \mathrm{~N}$ e $\pm 0,2 \%$ para $\delta^{13} \mathrm{C}$. Para o cálculo do valor isotópico de cada amostra foi aplicada a fórmula: $X$ amostra\%o $=[(R$ amostra $/ R$ referência) -1] x 1000; na qual $X=\delta^{15} \mathrm{~N}$ ou $\delta^{13} \mathrm{C} ; \mathrm{R}=\delta^{15} \mathrm{~N}: \delta^{14} \mathrm{~N}$ ou $\delta^{13} \mathrm{C}: \delta^{12} \mathrm{C}$. Os resultados foram expressos em partes por mil (\%o). Análise de variância (ANOVA) seguida do teste de Tukey a posteriori $(\alpha=0.05)$ no programa R versão 4.0.2 (R CORE TEAM, 2020) comparou a diferença das médias dos valores isotópicos ( $\delta 15 \mathrm{~N}$ e $\delta 13 C$ ) entre as espécies. Quando necessário, as variáveis foram transformadas para atender aos pressupostos dos testes paramétricos (linearidade, normalidade e homocedasticidade).

Na tabela 1 estão apresentados os dados de tamanho e peso do corpo das espécies analisadas, seu perfil isotópico e características gerais do hábito e modo de alimentação preferencial, de acordo com dados prévios de literatura [(LEGALL et al., 2020) para crustáceos; e (FROESE et al., 2019) para peixes]. O número amostral foi variável de acordo com a representatividade de cada espécie nos eventos de pesca. Genidens genidens se destacou das demais espécies com os valores mais pesados de $\delta^{15} \mathrm{~N}$ e $\delta^{13} \mathrm{C}$, enquanto 
Macrobrachium acanthurus apresentou os valores mais leves para ambos os isótopos (Tabela 1). ANOVA demonstrou diferenças significantes entre as espécies em relação ao perfil isotópico de nitrogênio (F=38,2, g.I. $=6, p<0.0001)$ e carbono $(F=24,3$, g.I. $=6, p<0.0001)$.

O teste de Tukey a posteriori destacou agrupamentos entre as espécies de acordo com os respectivos perfis isotópicos. Os valores de $\delta^{15} \mathrm{~N}$ permitiram agrupar Callinectes bocourti, M. acanthurus, Anchoviella lepidentostole e Leporinus copelandii com os valores mais leves, seguido de Lycengraulis grossidens e Centropomus undecimalis, e depois $G$. genidens, destacada das demais espécies com a média mais pesada de $\delta^{15} \mathrm{~N}$ (Figura 2). Em relação a $\delta^{13} \mathrm{C}$, os valores mais leves (mais negativos) se referem ao grupo formado por M. acanthurus, L. copelandii e C. undecimalis, depois C. bocourti com valor intermediário, e L. grossidens, A. lepidentostole e G. genidens com valores mais pesados de $\delta^{13} \mathrm{C}$ (menos negativos) (Figura 2).

Tabela 1: Caracterização dos crustáceos e peixes coletados no estuário interno do Rio Paraíba do Sul quanto ao tamanho e peso do corpo, perfil isotópico $\left(\delta^{15} \mathrm{~N}\right.$ e $\left.\delta^{13} \mathrm{C}\right)$ e características gerais do hábito e modo de alimentação. Os valores estão expressos como média \pm desvio padrão. $O$ tamanho do corpo de $C$. bocourti é medido como a largura da carapaça, e para as demais espécies como o comprimento total.

\begin{tabular}{|c|c|c|c|c|c|}
\hline $\begin{array}{l}\text { Espécie } \\
\text { Número amostral } \\
\text { (n) }\end{array}$ & $\begin{array}{l}\text { Tamanho } \\
(\mathrm{cm})\end{array}$ & $\begin{array}{l}\text { Peso } \\
\text { (g) }\end{array}$ & $\begin{array}{l}\delta^{15} \mathrm{~N} \\
(\%)\end{array}$ & $\begin{array}{l}\delta^{13} \mathrm{C} \\
(\% \circ) \\
(\%)\end{array}$ & Características gerais do hábito e modo de alimentação \\
\hline \multicolumn{6}{|l|}{ CRUSTÁCEOS } \\
\hline $\begin{array}{l}\text { Callinectes bocourti } \\
\text { (Milne-Edwards, } \\
\text { 1879) } \\
(n=5)\end{array}$ & $12,8 \pm 2,7$ & $\begin{array}{l}109,6 \pm \\
40,7\end{array}$ & $\begin{array}{l}11,9 \pm \\
0,5\end{array}$ & $\begin{array}{l}-20,1 \\
\pm 2,9\end{array}$ & $\begin{array}{l}\text { Demersal, estuarina e generalista que se alimenta de outros } \\
\text { crustáceos, moluscos, animais em decomposição e detritos }\end{array}$ \\
\hline $\begin{array}{l}\text { Macrobrachium } \\
\text { acanthurus } \\
\text { (Wiegmann, 1836) } \\
(\mathrm{n}=20)\end{array}$ & $12,2 \pm 2,2$ & $\begin{array}{ll}13,4 \quad \pm \\
8,0\end{array}$ & $\begin{array}{l}11,5 \pm \\
0,6\end{array}$ & $\begin{array}{l}-22,3 \\
\pm 1,6\end{array}$ & $\begin{array}{l}\text { Demersal, dulcícola e generalista que se alimenta de algas, } \\
\text { invertebrados, pequenos peixes, animais em decomposição } \\
\text { e detritos }\end{array}$ \\
\hline \multicolumn{6}{|l|}{ PEIXES } \\
\hline $\begin{array}{l}\text { Lycengraulis } \\
\text { grossidens } \\
\text { (Spix \& Agassiz, } \\
\begin{array}{l}1829) \\
(\mathrm{n}=15)\end{array}\end{array}$ & $15,8 \pm 4,3$ & $\begin{array}{ll}17,7 \quad \pm \\
6,7\end{array}$ & $\begin{array}{l}13,1 \pm \\
0,3\end{array}$ & $\begin{array}{l}-18,3 \\
\pm 0,9\end{array}$ & $\begin{array}{l}\text { Pelágica e anádroma que se alimenta de crustáceos } \\
\text { planctônicos, larva de insetos e pequenos peixes }\end{array}$ \\
\hline $\begin{array}{l}\text { Anchoviella } \\
\text { lepidentostole } \\
\text { (Fowler, 1911) } \\
(\mathrm{n}=9)\end{array}$ & $16,0 \pm 1,3$ & $\begin{array}{ll}23,7 & \pm \\
6,7 & \end{array}$ & $\begin{array}{l}11,8 \pm \\
0,5\end{array}$ & $\begin{array}{l}-18,9 \\
\pm 0,7\end{array}$ & $\begin{array}{l}\text { Pelágica e anádroma que se alimenta de larvas de crustáceos } \\
\text { e de outros invertebrados }\end{array}$ \\
\hline $\begin{array}{l}\text { Leporinus copelandii } \\
\text { (Steindachner, } \\
\begin{array}{l}\text { 1875) } \\
(\mathrm{n}=3)\end{array}\end{array}$ & $14,2 \pm 4,2$ & $\begin{array}{ll}34,1 & \pm \\
25,5\end{array}$ & $\begin{array}{l}11,8 \pm \\
0,3\end{array}$ & $\begin{array}{l}-21,9 \\
\pm 1,2\end{array}$ & $\begin{array}{l}\text { Bentopelágica, dulcícola e onívora que se alimenta de presas } \\
\text { animais pelágicas e demersais }\end{array}$ \\
\hline $\begin{array}{l}\text { Genidens genidens } \\
(\text { Cuvier, 1829) } \\
\underline{(\mathrm{n}=20)}\end{array}$ & $29,3 \pm 4,8$ & $\begin{array}{l}163,0 \pm \\
105,1\end{array}$ & $\begin{array}{l}14,2 \pm \\
0,4\end{array}$ & $\begin{array}{l}-17,7 \\
\pm 0,6\end{array}$ & Demersal e estuarina-marinha que se alimenta de zoobentos \\
\hline $\begin{array}{l}\text { Centropomus } \\
\text { undecimalis } \\
\text { (Bloch, 1792) } \\
(\mathrm{n}=13)\end{array}$ & $24,5 \pm 6,6$ & $\begin{array}{l}184,4 \pm \\
141,3\end{array}$ & $\begin{array}{l}13,2 \pm \\
1,2\end{array}$ & $\begin{array}{l}-21,5 \\
\pm 2,1\end{array}$ & $\begin{array}{l}\text { Anfídroma que se alimenta de crustáceos e peixes, podendo } \\
\text { ocupar ambiente demersal e pelágico de acordo com a fase } \\
\text { do ciclo de vida }\end{array}$ \\
\hline
\end{tabular}




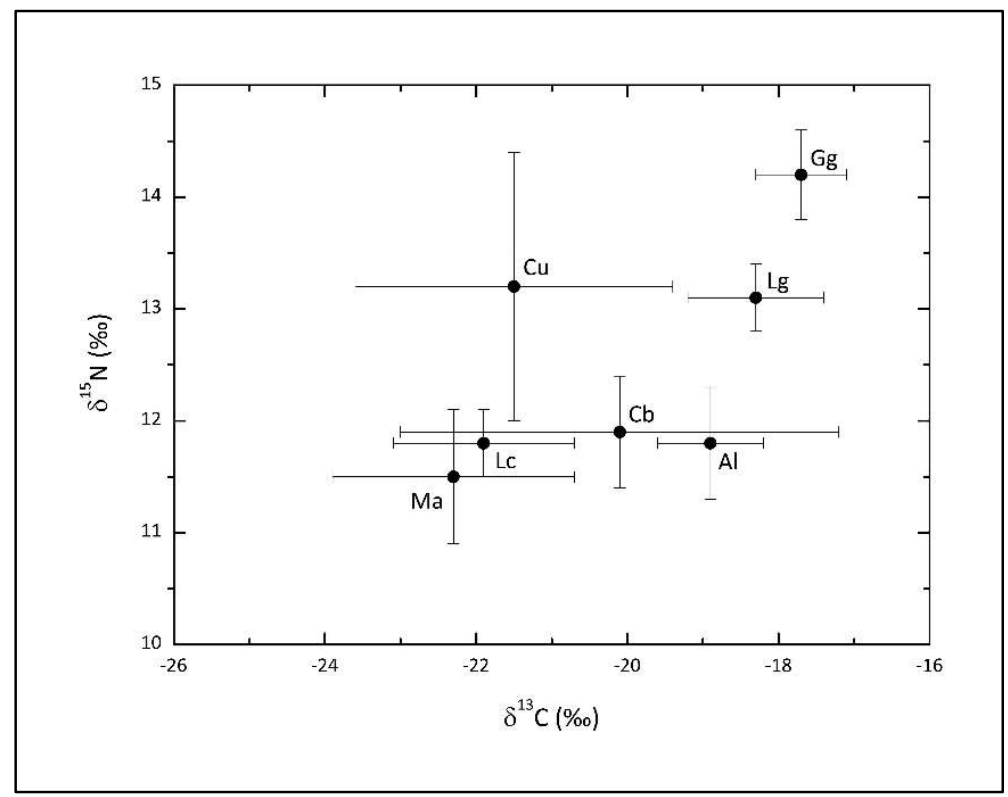

Figura 2: Média dos valores de $\delta^{15} \mathrm{~N}$ e $\delta^{13} \mathrm{C}$ dos crustáceos e peixes coletados no estuário interno do Rio Paraíba do Sul. As barras representam o desvio padrão. Ma: Macrobrachium acanthurus; Lc: Leporinus copelandii; Cu: Centropomus undecimalis; Cb: Callinectes bocourti; Al: Anchoviella lepidentostole; Lg: Lycengraulis grossidens e Gg: Genidens genidens.

\section{DISCUSSÃO}

Em geral, os perfis isotópicos dos crustáceos e peixes analisados apresentaram variações condizentes com sua posição trófica, medida por $\delta^{15} \mathrm{~N}$, e hábitat trófico, medido por $\delta^{13} \mathrm{C}$. Os valores mais pesados de $\delta^{15} \mathrm{~N}$ e $\delta^{13} \mathrm{C}$ em $\mathrm{G}$. genidens convergem com o perfil isotópico determinado para a espécie a partir de indivíduos capturados em área marinha costeira adjacente a foz secundária do Rio Paraíba do Sul (GATTS et al., 2020). Os valores também são próximos daqueles determinados em outras espécies de bagres (G. barbus e Bagre bagre) provenientes da pesca marinha costeira praticada na região (BENEDITTO et al., 2018). Dessa forma, o perfil isotópico da espécie se mantém constante na área de estudo, e as semelhanças entre os valores desse estudo e àqueles obtidos em Gatts et al. (2020) confirmam a ampla movimentação de $G$. genidens entre os ambientes estuarino e marinho.

Espécies que se alimentam de detritos orgânicos ou de presas situadas na base de cadeias alimentares, tanto bentônicas quanto pelágicas, normalmente tem valores de $\delta^{15} \mathrm{~N}$ mais leves, refletindo posição trófica inferior (FRY, 2008). Isso foi registrado para M. acanthurus, C. bocourti e A. lepidentostole. No entanto, os valores mais leves de $\delta^{15} \mathrm{~N}$ em L. copelandii quando comparado a $C$. undecimalis, L. grossidens e G. genidens não eram esperados devido às semelhanças no seu modo de alimentação preferencial (Tabela 1). Em geral, $\delta^{15} \mathrm{~N}$ é enriquecido em níveis tróficos superiores (FRY, 2008), mas esse isótopo também é sensível ao tamanho corporal dos consumidores, estado nutricional, metabolismo de excreção e diferenças quantitativas e qualitativas na ingestão alimentar, incluindo os valores isotópicos das suas presas (KURLE et al., 2001; JENNINGS et al., 2002; BENEDITTO et al., 2018). Dessa forma, a interpretação do perfil isotópico de $\delta^{15} \mathrm{~N}$ deve ser feita com cautela para definição das respectivas posições tróficas.

Os perfis isotópicos de $\delta^{13} \mathrm{C}$ demonstram o hábitat trófico das espécies (PETERSON et al., 1987; FRY, 2008). Nesse caso, espera-se que consumidores que se alimentam em diversos estratos da coluna d'água ( $L$. 
copelandii e $C$. undecimalis) ou que fazem uso de fontes alimentares de origens variadas (detritos, algas e animais) (C. bocourti e $M$. acanthurus) tenham desvios padrões mais elevados em comparação a consumidores que mantém maior constância no hábitat trófico (A. lepidentostole, L. grossidens e G. genidens), conforme observado nas espécies estudadas (Tabela 1). Os valores de $\delta^{13} \mathrm{C}$ mais leves (mais negativos) registrados em espécies exclusivamente dulcícolas ou estuarinas, tais como $C$. bocourti, $M$. acanthurus e L. copelandii podem refletir a influência do aporte de material de origem terrestre (plantas C3 e C4, por exemplo) decorrente da vazão do Rio Paraíba do Sul. Já os valores de $\delta^{13} \mathrm{C}$ mais pesados (menos negativos) registrados em A. lepidentostole, L. grossidens e G. barbus demonstram a influência marinha na sua alimentação, uma vez que são espécies de que se movimentam entre os diferentes ambientes. A condição anfídroma de $C$. undecimalis e o fato da espécie ter grande variação ontogenética quanto ao hábitat e hábito alimentar preferenciais, conforme indicado por um outro autor, poderia explicar a média de $\delta^{13} \mathrm{C}$ mais leve, com desvio padrão elevado.

\section{CONSIDERAÇÕES FINAIS}

Os resultados do perfil isotópico de crustáceos e peixes associados ao estuário interno do Rio Paraíba do Sul demonstram o dinamismo desse ambiente, que recebe influências continentais e marinhas e abriga espécies com hábitos bem distintos. Isso se reflete nos valores isotópicos de nitrogênio e carbono determinados no músculo dos indivíduos analisados. Esse estudo registra pela primeira vez o perfil isotópico dessas espécies na região, revelando peculiaridades da sua ecologia alimentar e corroborando, em muitos casos, as informações da literatura. O acompanhamento das variações desse perfil isotópico ao longo do tempo permitirá identificar possíveis alterações nas relações alimentares desses organismos e, consequentemente, possíveis alterações nos recursos disponíveis na região.

AGRADECIMENTOS: Ao Dr. Marcelo Gomes de Almeida pelas análises de isótopos estáveis nas amostras, ao Conselho Nacional de Desenvolvimento Científico e Tecnológico - CNPq (301.259/2017-8) e a Fundação Carlos Chagas Filho de Amparo à Pesquisa do Estado do Rio de Janeiro - FAPERJ (E-26/202.770/2017, E26/210.064/2018, E-26/210.844/2018) pelo financiamento à pesquisa.

\section{REFERÊNCIAS}

ARAÚJO, F. G.. Composição e estrutura da comunidade de peixes do médio e baixo rio Paraíba do Sul, RJ. Revista Brasileira de Biologia, v.56, n.1, p.111-126, 1996.

BIZERRIL, C. R.; PRIMO, P. B.. Peixes de águas interiores do estado do Rio de Janeiro. Rio de Janeiro: FEMAR, 2001.

BENEDITTO, A. P. M.; TAVARES, M. T. M.; MONTEIRO L. R. Isotopic niche of the catfishes Bagre bagre and Genidens barbus in a coastal area of southeastern Brazil. Biota Neotropica, v.18, n.3, e20180527, 2018. DOI: https://doi.org/10.1590/1676-0611-bn-2018-0527

FROESE, R.; PAULY, D.. FishBase. World Wide Web electronic publication, 2019

FRY, B.. Stable Isotope Ecology. New York: Springer Science Business Media, 2008.

GATTS, P. V.; FRANCO, M. A. L.; ALMEIDA, M. G.; ZALMON, I. R.; BENEDITTO, A. P. M.; COSTA, P. A. S.; REZENDE, C. E.. The trophic ecology of marine catfishes in south-eastern Brazil. Journal of the Marine Biological Association of the United Kingdom. v.100, n.1, p.133-142, 2020. DOI: https://doi.org/10.1017/S0025315419001164

JENNINGS, S.; PINNEGAR, J. K.; POLUNIN, N. V. C.; WARR, K. J.. Linking size-based and trophic analyses of benthic 
community structure. Marine Ecology Progress Series.

v.226, p.77-85, 2002. DOI:

https://doi.org/10.3354/meps226077

KRÜGER, G. C. T.; CARVALHO, C. E. V; FERREIRA, A. G.; GONÇALVES, G. M.; TRUCCOLO, E. C.; SCHETTINI C. A. F.. Dinâmica de carbono orgânico dissolvido no estuário do Rio Paraíba do Sul, R.J., sob diferentes condições de maré e descarga fluvial. Atlântica, v.25, n.1, p. 27-33, 2003.

KURLE, C. M.; WORTHY, G. A. J.. Stable isotope assessment of temporal and geographic differences in feeding ecology of northern fur seals (Callorhinus ursinus) and their prey.

Oecologia, v.126, p.254-265, 2001. DOI:

https://doi.org/10.1007/s004420000518
LEGALL, N.; POUPIN, J.. Internet - CRUSTA: Database of Crustacea (Decapoda and Stomatopoda), with special interest for those collected in French overseas territories. 2020

PETERSON, B.J.; FRY, B.. Stable isotopes in ecosystem studies. Annual Review of Ecology and Systematics. v.18, p.293-320, 1987. DOI:

https://doi.org/10.1146/annurev.es.18.110187.001453

R CORE TEAM. R: A language and environment for statistical computing. Viena: R Foundation for Statistical Computing, 2020.

A CBPC - Companhia Brasileira de Produção Científica (CNPJ: 11.221.422/0001-03) detém os direitos materiais desta publicação. Os direitos referem-se à publicação do trabalho em qualquer parte do mundo, incluindo os direitos às renovações, expansões e disseminações da contribuição, bem como outros direitos subsidiários. Todos os trabalhos publicados eletronicamente poderão posteriormente ser publicados em coletâneas impressas sob coordenação da Sustenere Publishing, da Companhia Brasileira de Produção Científica e seus parceiros autorizados. Os (as) autores (as) preservam os direitos autorais, mas não têm permissão para a publicação da contribuição em outro meio, impresso ou digital, em português ou em tradução. 